\title{
Cell culture-based spectral methods: how to prepare blanks?
}

\section{Opinion}

Divers tests that are routinely done in laboratories are based on spectral methods. ${ }^{1}$ Such methods are based on the biophysical interactions between the compounds (we are willing to evaluate the activity) and one or more electromagnetic waves of selected lengths. During these tests it is common to treat sample or cells, with a drug for example, the evaluate parameters such as cell viability, enzymatic activities and metabolites concentrations. In cell-based drug tests ${ }^{2}$ for example we incubate cells with different concentrations of drugs, including the control that is represented by cells incubate without the concerned drug. In addition, we also need a sample, cell culture medium without cells that represents the blank. The spectral values obtained from the blank are deduced from the other values (corresponding to the control and drug-treated samples) before we calculated the values we need (such as cell viability). However, do we need one blank or many blanks? Do we need to treat the blank or not?

These questions find its origin forms the fact that the spectral values (such as an absorbance during a cell viability test), may not due to the parameters we are willing to measure only but also to the drugs or to the molecular changes ${ }^{3}$ the compounds induce independently from the measured parameters. For instance, in cell viability tests. ${ }^{4,5}$ We measure absorbance that is correlated to the cell viability. The absorbance may be due not only to cell viability but also to other factors such as the spectral properties of the compounds, or to endogenous compounds that have been modified via a reaction with the studies compounds regardless of the cell viability. Therefore, we can obtain a higher absorbance, not due to higher cell viability but due to the absorbance of the studies compounds or the absorbance of other endogenous compounds that might have interacted with the studies compounds without affecting cell viability. A solution could be to use one blank for each drug concentration and add the same concentration of the drug to both the cells and the corresponding blank. Therefore, make sure the only difference in the spectral value (such as the absorbance) is due only to the cell viability.

This concept may be applied to the different cell culture-based and In vitro spectral methods in order to optimize the obtained results by reducing the chances of spectral interactions.
Volume 2 Issue 5 - 2015

\author{
Abdelaziz Ghanemi ${ }^{1,2,3}$ \\ 'Key Laboratory of Animal Models and Human Disease \\ Mechanisms of the Chinese Academy of Sciences \& Yunnan \\ Province, Kunming Institute of Zoology, China \\ 'University of Chinese Academy of Sciences, China \\ ${ }^{3}$ University of Chinese Academy of Sciences, China
}

Correspondence: Key Laboratory of Animal Models and Human Disease Mechanisms, Kunming Institute of Zoology Chinese Academy of Sciences, No. 32 Jiaochang Donglu, Kunming 650223, China, Email ghanemiabdelaziz@hotmail.com

Received: December 20, 2015 | Published: December 22, 2015

\section{Acknowledgements}

Abdelaziz Ghanemi is a recipient of a 2013 CAS-TWAS President's Postgraduate Fellowship.

\section{Conflict of interest}

The author declares no conflict of interest.

\section{References}

1. Cui H, Singh VP. Physica A: Statistical Mechanics and its Applications. 2016;442:91-99.

2. Ghanemi A. Cell cultures in drug development: Applications, challenges and limitations. Saudi Pharmaceutical Journal. 2015;23(4):453-454.

3. Ghanemi A. Biological properties and perspective applications of "Bioneuter" chemicals? Saudi Pharm J. 2014;22(1):1-2.

4. Yang Y, Lu Y, Wu QY, et al. Evidence of ATP assay as an appropriate alternative of MTT assay for cytotoxicity of secondary effluents from WWTPs. Ecotoxicol Environ Saf. 2015;122:490-496.

5. Stepanenko AA, Dmitrenko V. Pitfalls of the MTT assay: Direct and off-target effects of inhibitors can result in over/underestimation of cell viability. Gene. 2015;574(2):193-203. 